\title{
Design Loads for Wind Turbines using the Environmental Contour Method
}

\author{
Korn Saranyasoontorn* and Lance Manuel ${ }^{\dagger}$ \\ Dept. of Civil, Architectural and Environmental Engineering, University of Texas, Austin, TX 78712
}

\begin{abstract}
When interest is in establishing ultimate design loads for wind turbines such that a service life of say 20 years is assured, alternative procedures are available. One class of methods works by employing statistical loads extrapolation techniques following development first of ten-minute load maxima distributions (conditional on inflow parameters such as mean wind speed and turbulence intensity). The parametric conditional load distributions require extensive turbine response simulations over the entire inflow parameter range. We will refer to this first class of methods as the "parametric method." An alternative method is based on traditional structural reliability concepts and isolates only a subset of interesting inflow parameter combinations that are easily first found by working backward from the target return period of interest. This so-called inverse reliability method can take on various forms depending on the number of variables that are modeled as random. An especially attractive form that separates inflow (environmental) variables from turbine load/response variables and further neglects variability in the load variables given inflow is referred to as the Environmental Contour (EC) method. We shall show that the EC method requires considerably smaller amounts of computation than the parametric method. We compare accuracy and efficiency of the two methods in 1- and 20-year design out-of-plane blade bending loads at the root of two $1.5 \mathrm{MW}$ turbines. Simulation models for these two turbines with contrasting features, in that one is stall-regulated and the other pitch-regulated, are used here. Refinements to the EC method that account for the effects of the neglected response variability are proposed to improve the turbine design load estimates.
\end{abstract}

\section{Introduction}

$T^{\mathrm{N}}$ recent years, there has been an increased interest in employing probabilistic design approaches for design lof wind turbines against extreme limit states. Several different procedures have been suggested in studies by Madsen et al, ${ }^{1}$ Larsen et al, ${ }^{2}$ Ronold and Larsen, ${ }^{3}$ Fitzwater and Winterstein, ${ }^{4}$ Manuel et al,${ }^{5}$ Fitzwater et al, ${ }^{6}$ Moriarty et al, ${ }^{7,8}$ Peeringa, ${ }^{9}$ and Cheng et al. ${ }^{10}$ Many of these studies focus on how to model the distribution of turbine loads conditional on inflow random variables using parametric approaches. Such conditional load distributions are next integrated with the joint probability distribution of all probable environmental or inflow conditions to finally yield by extrapolation the desired long-term load associated with a given reliability level or probability of failure. Often, with parametric methods one obtains statistics of turbine loads (e.g., moments, maxima, etc.) conditional on inflow random variables; then, parametric distribution models (e.g., Weibull) are fit to load maxima and, finally, extrapolation to desired probability levels is carried out to yield design loads. Considerable amounts of turbine load simulation are usually necessary to estimate load statistics.

An alternative approach for establishing design loads associated with a target reliability involves the use of structural reliability techniques. The Environmental Contour (EC) method ${ }^{11}$ is one such approach that is especially easy to apply as well as efficient. It belongs to the class of inverse reliability techniques that work by searching a narrow subset of combinations of all important random variables that affect performance of the turbine. The EC method's simplicity is due to the fact that turbine load variability is approximated as having low variability (relative to the inflow random variables) and as such the search for the design load

\footnotetext{
* Graduate Research Assistant
}

${ }^{\dagger}$ Associate Professor 
amounts to a search for the largest "median" load conditional on inflow random variables. Besides, not all inflow random variable combinations (e.g., mean wind speed and turbulence intensity) need to be searched. Rather, only a subset of such combinations that guarantee the target reliability are of interest. The method is approximate for two reasons: (i) it ignores response variability as already pointed out; and (ii) it assumes linear limit state surfaces that separate the "safe" domain from the "unsafe" domain of random variables in the problem. Recently, Peeringa, ${ }^{9}$ Fitzwater et al, ${ }^{12}$ and Saranyasoontorn and Manuel ${ }^{13}$ demonstrated how the EC method could be applied to establish ultimate wind turbine blade bending design loads for various wind turbines.

Our interest here is in establishing design loads for commercial-sized wind turbines that are common today. Accordingly, two 1.5MW turbines will be our focus here. Stall- and pitch-regulated turbines have very different operating characteristics; here, one of each type will be considered in our examples, and attempts to compare the design loads and the important inflow variables that influence these loads will be made. Our focus here is on out-of-plane bending loads at the blade root and we will only consider failures in the operating range of wind speeds; for the turbines selected, this operating range goes from a cut-in speed of $5 \mathrm{~m} / \mathrm{s}$ to a cut-out wind speed of $25 \mathrm{~m} / \mathrm{s}$. Moriarty et al ${ }^{8}$ have employed parametric methods and statistical loads extrapolation to derive design loads for these same turbines using simulation; hence, their results will serve conveniently to verify the 20-year design loads that we will obtain with the EC method. Moreover, in the study by Moriarty et al, ${ }^{8}$ to validate their own results at the 1 -year level, they performed the equivalent of one entire year's simulation of turbine loads. Hence, with the EC method, we also derive 1-year design loads to compare with the extrapolated load estimates of Moriarty et al ${ }^{8}$ as well as the "exact" estimate directly obtained from their simulations. Note that our expectation is that the pitch-regulated turbine design loads will be somewhat harder to predict because of the more complex control system associated with it. A parametric model for the distribution of (short-term) ten-minute load maxima carried out by Moriarty et $a{ }^{8}{ }^{8}$ for instance, was unsuccessful in accurately predicting design loads for the pitch-regulated turbine. Similar difficulties were not seen with the stall-regulated turbine.

Because the EC method is based on the use of the median turbine load (conditional on inflow variables) and as such ignores variability in turbine response/loads, improvements have been suggested in the literature including the use of higher-than-median fractiles of the load derived using "omission factors" 14 or the use of "inflated contours" associated with larger return periods than the target. Both these refinements depend on additional simulations performed to account for response variability locally. Here, a different approach that starts with the design load derived from the EC method is used along with a "local" fit to a chosen distribution (such as the lognormal) for the conditional load. The improvement in accuracy of applying this refinement to the EC method is discussed.

\section{Reliability-Based Design for a Target Return Period}

\section{A. Design Loads for a Target Return Period}

Reliability-based design for wind turbines against ultimate limit states is concerned with establishing the appropriate load level for design so as to guarantee a specified maximum target probability of failure. Suppose, for example, that one is interested in a $T$-year service life for the turbine or, equivalently, the $T$-year return period load. Based on the assumption of stationarity of inflow conditions in segments of 10-minute duration, it is sufficient to estimate the design load, $L_{T}$ by ensuring that the extreme load in ten minutes , $L_{10 \text { min }}$, will exceed $L_{T}$ with a probability consistent with the service life or return period, $T$. For the sake of illustration, assume that the prescribed value of $T$ is 20 years. Since there are $20 \times 365 \times 24 \times 6=1,051,200$ (independent) 10-minute segments in twenty years, the failure probability (i.e., the probability that $L_{10 \mathrm{~min}}$ exceeds $L_{T}$ in ten minutes should be no greater than $p_{f}=1 / 1,051,200=9.513 \times 10^{-7}$. Note that this is a "short-term" failure probability since it refers to the probability of failure in a segment of 10-minute duration.

It is common to establish probabilistic distributions for inflow parameters such as the 10-minute mean, $V$, and standard deviation, $\sigma$, of the hub-height wind speed. The design load, $L_{T}$ needs to be derived for the probability $p_{f}$ above and by considering all possible values of $V$ and $\sigma$ and their joint probabilities of occurrence. This can be done by adjusting $L_{T}$ in the equation below until the probability on the left-hand side equals $p_{f}$. 


$$
P\left[L_{10 \min }>L_{T}\right]=\int_{0}^{\infty} \int_{0}^{\infty} P\left[L_{10 \min }>L_{T} \mid v, \sigma\right] f_{V, \sigma}(v, \sigma) d \sigma d V
$$

where $f_{V, \sigma}(v, \sigma)$ represents the joint probability density function (PDF) of the inflow (environmental) variables. This PDF is site-specific. Computation of the integral on the right-hand side of Eq. (1) for different values of $L_{T}$ is expensive and involves numerous computations of the short-term conditional probability, $P\left[L_{10 \min }>L_{T} \mid v, \sigma\right]$. Hence, several alternatives exist for establishing the $T$-year load, $L_{T}$. One of these involves systematically computing $P\left[L_{10 \mathrm{~min}}>L_{T} \mid v, \sigma\right]$ or a variation of such short-term conditional loads (sometimes using local maxima of the load time history instead of extremes) by the use of parametric distributions fit to statistical moments, peaks over specified thresholds, etc. This "parametric method" takes no account of any specific inflow variable combinations that are more interesting from the point of view of causing large loads. The parametric method requires extensive turbine response simulation over the entire range of the inflow variable space. Several studies employing the parametric method to establish turbine design loads for ultimate limit states have been proposed. Ronold et al ${ }^{15}$ proposed a second-order polynomial model for turbine load statistical moments. Veers and Winterstein ${ }^{16}$ proposed a power-law parametric model which was adopted in other studies..$^{5,7}$ Recently, Moriarty et $\mathrm{al}^{8}$ developed a parametric model that accounts for the highly nonlinear behavior of the statistical moments of the extreme load response, especially near the rated wind speed of the pitch-regulated turbine studied there.

Note that in Eq. (1) $f_{V, \sigma}(v, \sigma)$ is site-specific, while $P\left[L_{10 \mathrm{~min}}>L_{T} \mid v, \sigma\right]$ is turbine-specific. Accordingly, a second method for establishing turbine design loads for a specified return period, $T$, is based on separating the environmental variability from the (conditional) turbine load variability given environmental conditions. This method (called the Environmental Contour method) is based on structural reliability principles and can yield the $T$-year design load with far less computational effort than is involved with the use of parametric methods and computations of integrals as in Eq. (1).

\section{B. Environmental Contour Method}

The Environmental Contour (EC) method was developed by Winterstein et $\mathrm{al}^{11}$ and has been used extensively to derive design loads for fixed and floating offshore oil platforms. Details related to the theoretical framework for the application of the method can be found elsewhere. ${ }^{11,13,17}$ Since the method is derived from the classical First-Order Reliability Method (FORM), it assumes that in the space of the random variables affecting performance of the structure under consideration, the safe and unsafe regions are separated by a linear limit state hyperplane. Also, the EC method only considers the environmental variables as random; a contour associated with the desired probability of failure, $p_{f}$, is constructed, and the largest "median" extreme load with different environmental variables on the $p_{f}$ contour is sought. This defines the design load.

Suppose, as we have here, that the only environmental random variables are the 10-minute mean, $V$, and standard deviation, $\sigma$ of the hub-height wind speed. These are usually jointly distributed random variables. It is convenient, in the EC method, to consider independent standard normal random variables, $U_{1}$ and $U_{2}$, that can be mapped to $V$ and $\sigma$ using the Rosenblatt transformation. ${ }^{18}$ Associated with the probability of failure, $p_{f}$, defined before in terms of the return period, $T$, it is easy to construct the "environmental contour" in $\boldsymbol{U}$-space which represents all points on the circle such that $u_{1}^{2}+u_{2}^{2}=\beta_{T}^{2}$ where $\beta_{T}$ is such that $\Phi\left(-\beta_{T}\right)$ is equal to $p_{f}$.

Thus, given $F_{V}(v)$, the Cumulative Distribution Function $(\mathrm{CDF})$ of $V$ and $F_{\sigma \mid V}(\sigma \mid v)$, the CDF of $\sigma$ conditional on $V$, the circle in $\boldsymbol{U}$-space can be transformed to the contour in physical $\boldsymbol{X}$ space or $V-\sigma$ space using the Rosenblatt transformation. ${ }^{18}$

$$
\Phi\left(u_{1}\right)=F_{V}(v) ; \quad \Phi\left(u_{2}\right)=F_{\sigma \mid V}(\sigma \mid v)
$$

In the numerical studies that follow, we will employ the EC method to derive out-of-plane bending design loads (at blade root) for two 1.5MW wind turbines. Our focus is primarily on 20-year design loads. These loads will be compared with those derived by Moriarty et $\mathrm{al}^{8}$ for the same turbines. For the sake of comparison, 1-year design loads will also be studied. 


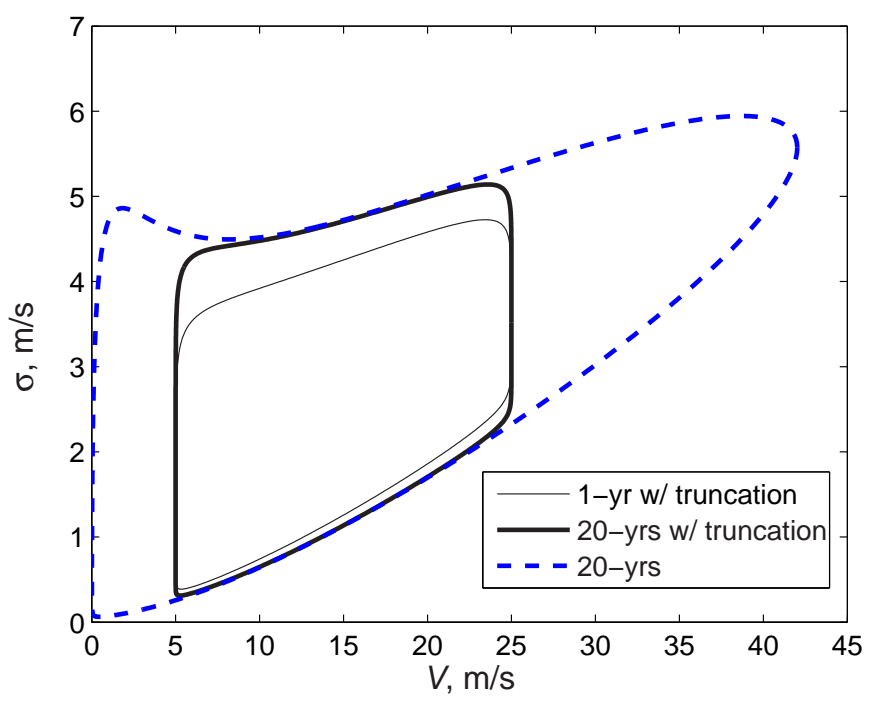

Figure 1. Environmental contours corresponding to 1- and 20-year return periods. (The 20-year contour is also shown before truncation below cut-in and above cut-out wind speeds.)

\section{Numerical Examples}

\section{A. Environment}

Consider a site where per IEC guidelines, ${ }^{19}$ the 10 -minute mean wind speed, $V$ is assumed to follow a Rayleigh distribution. Also, assume that the standard deviation of the wind speed, $\sigma$, conditional on $V$ follows a lognormal distribution. Thus, we have:

$$
\begin{gathered}
f_{V}(v)=\frac{2 v}{\alpha^{2}} \exp \left[-\left(\frac{v}{\alpha}\right)^{2}\right] ; \quad \alpha=\frac{2 \mu_{V}}{\sqrt{\pi}} \\
f_{\sigma \mid V}(\sigma)=\frac{1}{\sqrt{2 \pi \sigma \zeta}} \exp \left[-\frac{1}{2}\left(\frac{\ln \sigma-\lambda}{\zeta}\right)^{2}\right]
\end{gathered}
$$

where $\mu_{V}$ is the mean value of the 10-minute average wind speed at hub height, while $\lambda$ and $\zeta$ depend on the value of $V$ and are functions of $I_{15}$, the characteristic value of the turbulence intensity at $15 \mathrm{~m} / \mathrm{s}$, and $a$, a slope parameter defined in the IEC guidelines. ${ }^{19}$ For a Class IA turbine site, which is what we will consider here for both turbines, $\mu_{V}$ is $10 \mathrm{~m} / \mathrm{s}, I_{15}$ is 0.18 , and $a$ is 2 . Expressions for the parameters $\lambda$ and $\zeta$ in Eq. (4) are given in the Appendix.

Environmental contours corresponding to a 20-year return period are shown with the dotted contour in Fig. 1. If we are interested only in design loads corresponding to the operating range of power production, namely between cut-in and cut-out wind speeds of $5 \mathrm{~m} / \mathrm{s}$ and $25 \mathrm{~m} / \mathrm{s}$, respectively, the probability of failure, the PDF given by Eq. 3 needs to be truncated. Moreover, the probability of failure, $p_{f}$, associated with the return period needs to be corrected to account for the fraction of time when the turbine is operating (versus when the wind speeds are below cut-in or above cut-out). Adjusting for this effect, environmental contours were derived for 1- and 20-year return periods and are shown with the light and dark solid lines, respectively, in Fig. 1. An expression for the truncated cumulative distribution function of $V$ is given in the Appendix.

\section{B. Description of the Turbines}

Two commercial-sized 1.5MW 3-bladed horizontal-axis wind turbines are considered here - the first is a pitch-regulated turbine developed as part of the WindPACT study ${ }^{20}$ while the second is a typical but generic stall-regulated turbine. Simulation models for the two turbines are the same as those employed in the study on design loads by Moriarty et al. ${ }^{8}$ Each turbine has a hub height of $84 \mathrm{~m}$, rotor diameter of $70 \mathrm{~m}$, and rated power of $1.5 \mathrm{MW}$. As discussed before, the turbines are assumed to have a cut-in wind speed $v_{i}$ of 5

$$
4 \text { of } 13
$$


$\mathrm{m} / \mathrm{s}$ and a cut-out speed $v_{o}$ of $25 \mathrm{~m} / \mathrm{s}$. The stall-regulated turbine has a constant speed of $16.9 \mathrm{RPM}$, while the pitch-regulated, variable speed machine has a rated speed of 20.4 RPM. The rated wind speeds of the stall- and pitch-regulated machines are $14 \mathrm{~m} / \mathrm{s}$ and $11 \mathrm{~m} / \mathrm{s}$, respectively. The EC method can be directly applied now that the environmental contours shown in Fig. 1 are available. However, in order to obtain the "short-term" median extreme response conditional on any combination of $V$ and $\sigma$ values that occurs on the contours, it is necessary that turbine response simulations be carried out. The two turbines are quite different in performance characteristics; it is anticipated that near the rated wind speed, non-monotonic changes in loads with wind speed are likely in the case of the pitch-regulated turbine. Hence, the contrasting turbines will make comparisons of results between the two types interesting.

\section{Design Loads using the EC Method}

In the following, let us consider the 20-year out-of-plane bending (OOPB) loads at the blade root of the two turbines. Preliminary trial searches along the 20-year environmental contour of Fig. 1 include evaluation of thirteen environmental inflow variable combinations (wind conditions). The mean wind speed, $V$, for these cases ranges from about $5 \mathrm{~m} / \mathrm{s}$ to $25 \mathrm{~m} / \mathrm{s}$ and corresponds to uniform separations in the tangential direction of the 20-year return period contour in standard normal $\boldsymbol{U}$ as shown in Fig. 2, Note that Fig. 2 may be directly mapped to the truncated 20-year contour of Fig. 1] according to Eq. 2, Preliminary turbine response simulations will only be performed for these thirteen cases which were selected because they are in the range of wind conditions where both $V$ and $\sigma$ can be high and, hence, can likely lead to large OOPB extremes. (In fact, we shall see later that for the pitch-regulated turbine, the maximum load is generally most likely to occur near the rated wind speed rather than near the cut-out speed where $V$ is largest.) For each wind condition combination, five 10-minute simulations are conducted with different random number seeds using SNwind $^{21}$ for the inflow turbulence field simulation and $\mathrm{FAST}^{22}$ for the turbine loads simulation. (We shall discuss how the number of simulations was selected later.) The median extreme from the five individual simulation extreme OOPB loads is extracted for each of the thirteen inflow variable combinations. Results from the search for the maximum OOPB extreme load from the thirteen combinations on the 20-year return period contour are summarized in Table 1 where the angle, $\phi$, for each combination searched in $\boldsymbol{U}$-space is illustrated in Fig. 2. Table 1 shows values of $V$ and $\sigma$ for each point in the search as well as median extreme OOPB loads. This preliminary search yields design OOPB loads of 3,092 and 2,217 kN-m, respectively, for the stall-regulated and pitch-regulated turbines. It is worth noting that the preliminary design load estimates for the two machines are quite different. The median extreme values as a function of the angle, $\phi$, are also plotted in Fig. 3 to verify that no abrupt changes or steep gradients in the median extreme loads occur with crude 11.25-degree steps in the angle, $\phi$, along the 20-year return period contours. The flowchart shown in Fig. 4 summarizes the procedure for establishing the design loads used for this example. The values of $N$ and $M$ referred to in the flowchart are 13 and 5, respectively, in the turbine load studies discussed here. Note that because of the contrasting wind turbine blade dynamics, one might have anticipated that the blade bending loads would reach their largest values near the cut-out wind speed $(25 \mathrm{~m} / \mathrm{s})$ and the rated wind speed (11 $\mathrm{m} / \mathrm{s}$ ), respectively, for the stall- and pitch-regulated machines. These anticipated findings may be confirmed from the design loads and associated inflow conditions summarized in Table 1. In fact, this intuition might in general make it possible to focus the search for the largest extreme load over a smaller region of the random variable space than was employed here. This would significantly reduce the computational effort involved in inflow turbulence and load simulation.

After the preliminary search, refinements of the design loads summarized in Table 1 are sought by performing additional turbine simulations for inflow condition combinations close to the "design point" on the 20-year return period contour. Four additional points on the contour are used to compute additional median extreme OOPB loads. In general, a larger number of simulations (than five) at each inflow variable combination, $V$ and $\sigma$, could be considered to obtain more accurate median extreme loads. However, here, we still use 5 simulations at each wind condition in this refinement following the preliminary search calculations. We show later that 5 simulations per wind condition is sufficient here in order to obtain accurate estimates of the ten-minute median extreme OOPB load. Results show that the new OOPB design load for the stallregulated is unchanged implying that the maximum load was found before the refinements, while the design load for the pitch-regulated turbine increased from 2,217 to $2,272 \mathrm{kN}-\mathrm{m}$. Thus, only very small changes (no greater than a $5 \%$ increase) in the design loads are found when the search is refined indicating a fairly stable solution. Results from the preliminary search might have been sufficient for this OOPB load for either of the two turbines. In passing, we note that the wind speed corresponding to the maximum load for the 


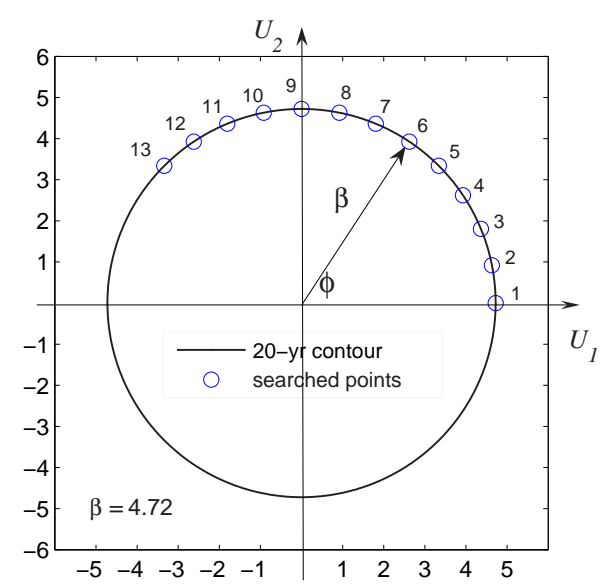

Figure 2. Environmental contour corresponding to a 20-year return period in standard normal space along with the thirteen points on the contour used to search for the maximum ten-minute OOPB load.

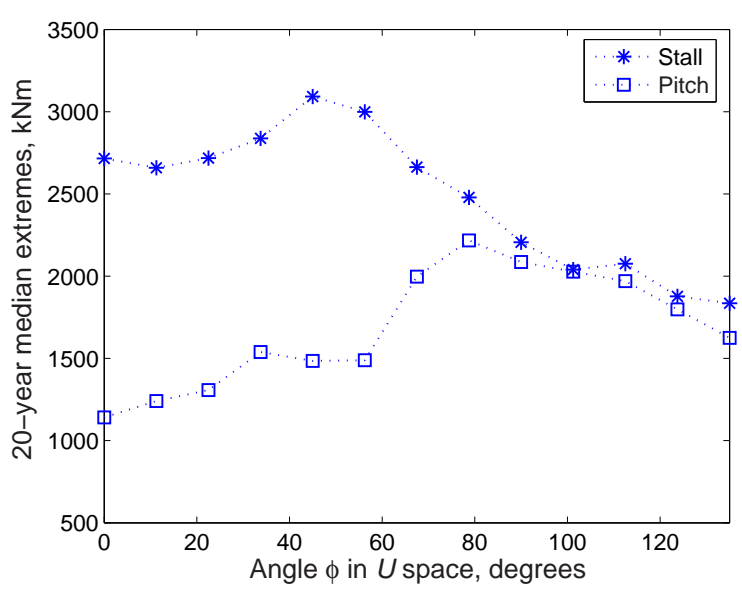

Figure 3. Estimated 20-year median extreme OOPB loads for the stall-regulated and pitch-regulated turbines for thirteen different wind conditions ( $V$ ranged from 5 to $25 \mathrm{~m} / \mathrm{s}$ and $\sigma$ ranged from 3.0 to $5.1 \mathrm{~m} / \mathrm{s}$ ).

Table 1. Estimates of the 20-year median extreme out-of-plane bending (OOPB) loads at the blade root of the $1.5 \mathrm{MW}$ stallregulated and pitch-regulated turbines after the preliminary search (along with associated inflow conditions).

\begin{tabular}{ccccc}
\hline$\phi$ & $V$ & $\sigma$ & \multicolumn{2}{c}{ Loads $(\mathrm{kN}-\mathrm{m})$} \\
\cline { 4 - 5 } (degrees) & $(\mathrm{m} / \mathrm{s})$ & $(\mathrm{m} / \mathrm{s})$ & Stall & Pitch \\
\hline 0.00 & 25.0 & 3.5 & 2,716 & 1,141 \\
11.25 & 25.0 & 3.9 & 2,659 & 1,241 \\
22.50 & 25.0 & 4.2 & 2,718 & 1,307 \\
33.75 & 25.0 & 4.6 & 2,838 & 1,539 \\
45.00 & 24.9 & 4.9 & $\mathbf{3 , 0 9 2}$ & 1,485 \\
56.25 & 24.0 & 5.1 & 2,999 & 1,489 \\
67.50 & 20.6 & 5.0 & 2,663 & 1,997 \\
78.75 & 15.5 & 4.7 & 2,479 & $\mathbf{2 , 2 1 7}$ \\
90.00 & 10.6 & 4.5 & 2,206 & 2,086 \\
101.25 & 7.1 & 4.4 & 2,040 & 2,027 \\
112.50 & 5.4 & 4.1 & 2,076 & 1,970 \\
123.75 & 5.1 & 3.7 & 1,877 & 1,798 \\
135.00 & 5.0 & 3.0 & 1,835 & 1,625 \\
\hline
\end{tabular}

6 of 13 


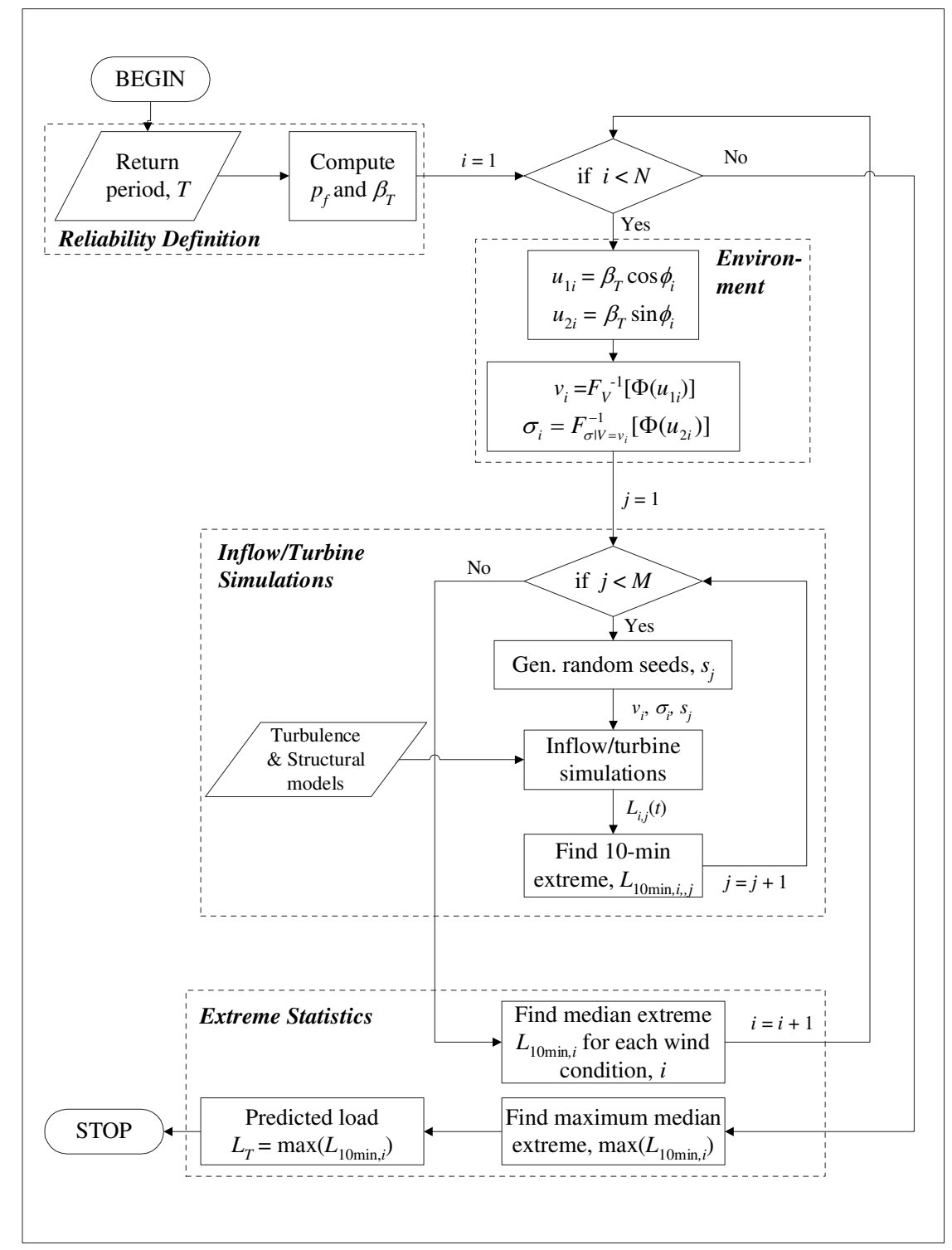

Figure 4. Flowchart for deriving a $T$-year design load using the Environmental Contour (EC) method. ( $N$ is the number of wind conditions used in the search for the maximum extreme load; $M$ is the number of simulations for each wind condition.) 


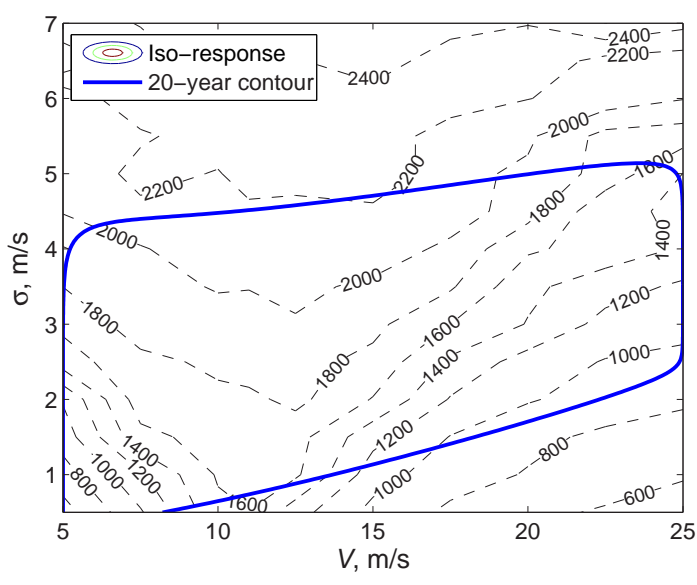

(a) Pitch-regulated

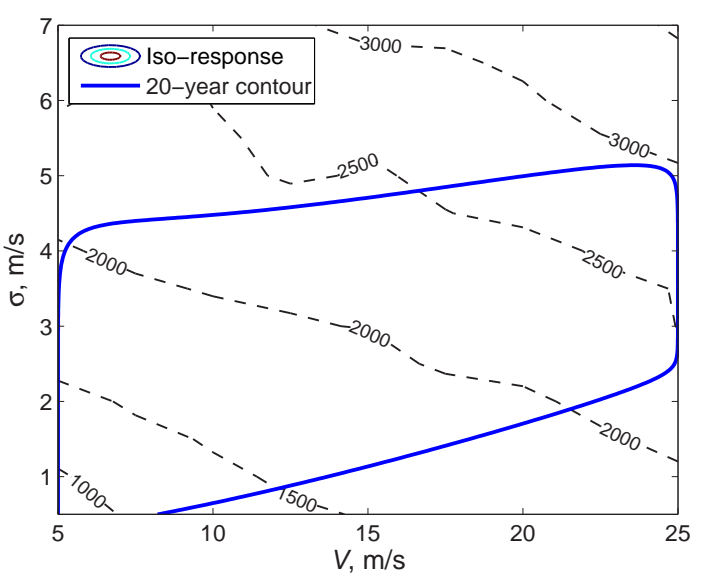

(b) Stall-regulated

Figure 5. 20-year environmental contours and OOPB load iso-response lines for the stall-regulated and pitchregulated turbines.

pitch-regulated turbine is $17.2 \mathrm{~m} / \mathrm{s}$, somewhat higher than the rated wind speed of $11 \mathrm{~m} / \mathrm{s}$. The EC method identifies this critical case and does not rely on merely the load associated with the rated wind speed for this turbine.

Because the behavior of the OOPB loads for a pitch-regulated machine can be complicated due to the control systems involved, a question that may arise is whether it is at all possible that the non-monotonic characteristics of the blade loads with wind speed could cause a larger extreme load for a point inside the $T$-year return period environmental contour. This issue has been raised ${ }^{9}$ as a possible criticism of the EC method and the suggestion is that perhaps less severe inflow variable combinations (those associated with a smaller return period than $T$ years) might cause larger loads than are found from combinations on the $T$-year contour itself. To address this issue, turbine response simulations are run over a range of wind speeds, $V$, between 5 and $25 \mathrm{~m} / \mathrm{s}$ (in $2.5 \mathrm{~m} / \mathrm{s}$ increments) as well as at the rated wind speed of the turbine $(11 \mathrm{~m} / \mathrm{s})$, and over a range of $\sigma$ values between 0.5 and $7 \mathrm{~m} / \mathrm{s}$ (in $0.5 \mathrm{~m} / \mathrm{s}$ increments). Five simulations per wind condition are performed and the median extreme OOPB loads at all the grid points (inflow variable combinations) are then used to construct median extreme iso-response curves for the OOPB load for both turbines. These are shown in Fig. 5] along with the 20-year return period environmental contour. It can be seen, for the pitch-regulated turbine, that even though the large OOPB loads are concentrated near the rated wind speed, the maximum value still occurs right on the 20-year contour, not inside it. This largest OOPB load for this turbine can be seen to be slightly greater than $2,200 \mathrm{kN}-\mathrm{m}$ (compared with the 2,217 $\mathrm{kN}-\mathrm{m}$ design load from our preliminary search described earlier). Note that the iso-response curves are obtained using a rather coarse grid (with a wind speed interval of $2.5 \mathrm{~m} / \mathrm{s}$ ); the earlier results from the refined search are likely to be more accurate than those summarized in Fig. 5. This brief discussion is meant to suggest acceptability of the EC method for use in design purposes for pitch-regulated wind turbines. Iso-response lines for the stall-regulated turbine also shown in Fig. 5 have far smoother trends with variation in inflow variables; no difficulties were expected by applying the EC method to predict design loads for this turbine. The 20-year OOPB design load for the stall-regulated machine based on this figure can be seen to be close to 3,000 kN-m (compared with the $3,092 \mathrm{kN}-\mathrm{m}$ design load from our preliminary search described earlier).

\section{Correction for response variability}

The EC method, as presented thus far, neglects turbine response variability conditional on inflow random variables. If this variability is indeed small, the EC method's design load estimates based on the median tenminute extreme OOPB load will be accurate. If, however, response variability is large, the search outlined above to yield the maximum median extreme will be inaccurate and unconservative. The error in predicted loads from the EC method is sensitive to the uncertainty of the "short-term" response variable, $L_{10 m i n}$, conditional on the environmental variables, $V$ and $\sigma$. Hereinafter, $L_{10 \text { min }}$ will be referred to as $L$ for brevity. 
If the response variable, $L$, has large variability associated with it, by using only the median extreme response, $\hat{L}$, the EC method could wrongly predict the long-term (20-year) extreme load. Several approaches have been proposed to account for the omitted response variability. These include the use of higher-than-median fractiles of the response conditional on environmental variables, and the use of an adjusted higher-than- $T$ return period contour for the $T$-year load. These methods rely on additional computations of gradients of the limit state function (and, thus, additional turbine response simulations) as well as evaluation of omission sensitivity factors. Saranyasoontorn and Manuel ${ }^{13}$ employed omission factors to numerically compute local gradients of the limit state function near the EC design point for wind turbine applications. Higher-thanmedian fractiles of the response variable (conditional on environmental variables) were then used to refine the design load based on the EC method. Such an approach, though shown to be efficient in providing results close to the "exact" values ${ }^{13}$ (that were computed independently by numerical integration of Eq. 1 over all random variables), still requires considerable additional computation. Accordingly, here we adopt another approach for correction for the omitted response variability that was presented by Winterstein and Engebretsen $^{17}$ for applications to two different offshore floating platforms. There, the neglected response variability in the EC method is identified as arising from two sources: (i) "background" variability in the median response extreme $\hat{L}$ (in our case, this is variability in the median level with changing wind conditions) and (ii) "response" variability (arising due to variability in simulated turbine response extremes at a given wind condition). In other words, the short-term extreme response variable can be expressed as $L=\hat{L} \cdot \epsilon{ }^{17}$ where $\hat{L}$ is the median extreme response as defined before while $\epsilon$ is a unit-median random variable. The latter reflects variability in the extreme response arising due to different simulated input turbulence time histories for any single environmental variable combination. Both variabilities can be accounted for by local lognormal distribution fits for the random variables, $\hat{L}$ and $\epsilon$, which then yield information on the variability of $L$. The local fits are made using ten-minute extreme response data at and near the EC method's initial design point. Standard deviations of the two transformed random variables (parameters of the lognormal distributions), $\ln \hat{L}$ and $\ln \epsilon$, are given as follows ${ }^{17}$

$$
\sigma_{\ln \hat{L}}=\frac{\ln \left(\hat{L}_{T_{1}} / \hat{L}_{T_{2}}\right)}{\beta_{T_{1}}-\beta_{T_{2}}} ; \quad \sigma_{\ln \epsilon}=\frac{\ln \left(\epsilon_{p_{1}} / \epsilon_{p_{2}}\right)}{\Phi^{-1}\left(p_{2}\right)-\Phi^{-1}\left(p_{1}\right)}
$$

where $T_{1}$ is the prescribed return period of interest; $T_{2}$ is a slightly shorter return period (associated with a slightly higher failure probability); $\beta_{T_{1}}$ and $\beta_{T_{2}}$ are related to the target failure probabilities associated with return periods, $T_{1}$ and $T_{2}$, as discussed earlier; $\epsilon_{p_{1}}$ and $\epsilon_{p_{2}}$ represent two fractiles of the response conditional on inflow variables; and $\Phi^{-1}()$ represents the standard normal inverse CDF. After the "background" and

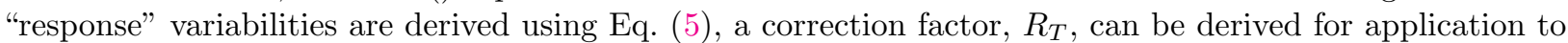
the $T$-year load as derived from the EC method may. Based on the local lognormal distribution assumptions, $R_{T}$, is given as follows: ${ }^{17}$

$$
R_{T}=\frac{L_{T}}{\hat{L}_{T}}=\exp \left[\left(\sigma_{\ln L}-\sigma_{\ln \hat{L}}\right) \beta_{T}\right]
$$

where $\sigma_{\ln L}^{2}=\sigma_{\ln \hat{L}}^{2}+\sigma_{\ln \epsilon}^{2}$. The use of this correction factor to the OOPB design loads based on the EC method for the two turbines is discussed next.

Table 2. 20-year median extreme estimates for the OOPB loads of the stall- regulated and pitch-regulated machines from first search, second search, and after correcting response variability. The final design loads found are 3,156 and 2,326 kN-m respectively for stall-

\begin{tabular}{|c|c|c|c|c|c|c|c|}
\hline & & \multicolumn{3}{|c|}{ Stall } & \multicolumn{3}{|c|}{ Pitch } \\
\hline & & $\begin{array}{c}V \\
(\mathrm{~m} / \mathrm{s})\end{array}$ & $\begin{array}{c}\sigma \\
(\mathrm{m} / \mathrm{s}) \\
\end{array}$ & $\begin{array}{c}\text { Load } \\
(\mathrm{kN}-\mathrm{m})\end{array}$ & $\begin{array}{c}V \\
(\mathrm{~m} / \mathrm{s})\end{array}$ & $\begin{array}{c}\sigma \\
(\mathrm{m} / \mathrm{s})\end{array}$ & $\begin{array}{c}\text { Load } \\
(\mathrm{kN}-\mathrm{m})\end{array}$ \\
\hline \multirow{2}{*}{$\mathrm{EC}$} & preliminary & 24.9 & 4.9 & 3,092 & 15.5 & 4.7 & 2,217 \\
\hline & refined & 24.9 & 4.9 & 3,092 & 17.2 & 4.8 & 2,272 \\
\hline $\mathrm{EC}^{+}$ & with response variability & 24.9 & 4.9 & 3,156 & 17.2 & 4.8 & 2,326 \\
\hline
\end{tabular}
and pitch-regulated machines. 
To account for neglected response variability in the 20-year loads derived using the EC method, we fit lognormal models to median response extremes, $\hat{L}$, at two different return periods -20 and 16 years, associated with reliability indices, $\beta_{16}$ and $\beta_{20}$, of 4.68 and 4.72 , respectively. Note that the differences between these two reliability index values, $\beta$, is small and, hence, we can use them to estimate the parameter, $\sigma_{\ln \hat{L}}$, of our local lognormal distribution that addresses "background" variability. For the environmental variables corresponding to the 20-year load, we also fit a lognormal model for $\epsilon$ using the $50 \%$ and $83 \%$ fractiles resulting from the response simulations at the design point from the EC method. An estimate of the parameter, $\sigma_{\ln \epsilon}$, of our local lognormal distribution that addresses "response" variability is thus obtained. These computations are carried out for both the stall- and the pitch-regulated turbines.

For the stall-regulated turbine, the logarithmic standard deviations, $\sigma_{\ln \hat{L}}$ and $\sigma_{\ln \epsilon}$, are 0.3431 and 0.0547 , respectively. This increases the design loads from 3,092 to $3,156 \mathrm{kN}-\mathrm{m}$ (only about a $2 \%$ increase). For the pitch-regulated turbine, $\sigma_{\ln \hat{L}}$ and $\sigma_{\ln \epsilon}$ are 0.584 and 0.077 , respectively. This again increases the design load very slightly (by approximately $2 \%$ ) from 2,272 to $2,326 \mathrm{kN}-\mathrm{m}$. It is worth noting that the "response" variability related to $\sigma_{\ln \epsilon}$ is rather small. This implies that, for the turbine and loads considered here, only a small number of simulations at each wind condition are needed to obtain stable response statistics. We will revisit this issue of uncertainty in the median extreme OOPB loads. A summary of the 20-year OOPB design loads of the two turbines is provided in Table 2 .

\section{E. Uncertainty in median extreme estimates from simulation}

An important concern in estimating load extremes from a limited number of simulations is that uncertainty in such estimates can be large. The EC method relies on the ability to have stable estimates from simulation at different trial points corresponding to different inflow conditions. The greater the uncertainty in the turbine load extremes, the greater will be the number of simulations needed. For instance, Moriarty et $\mathrm{al}^{8}$ studied the uncertainty in statistical moments of turbine loads and found that for the two turbines, at least thirty simulations per wind condition were required to provide stable statistical moments of local load maxima. This was especially true when higher moments such as skewness had to be estimated. With the EC method, results from the refinements based on the use of a locally fit lognormal model (for load extremes) discussed earlier suggest small variability in the OOPB median extremes. This can be claimed due to the small value of $\sigma_{\ln \epsilon}$ that was found earlier for both turbines. Here, we further investigate the uncertainty in median extreme estimates of the out-of-plane bending (OOPB) loads based on 100 ten-minute simulations. The bootstrap approach ${ }^{23}$ is used here to provide some insight into the uncertainty in the median extreme estimates close to, but not exactly at, the 20-year design point from the EC method. A total of 100 turbine simulations are generated that result in 100 OOPB extremes for our analysis. The bootstrap method is then employed to resample OOPB extremes with replacement 1000 times. The mean values of the 1000 resampled median OOPB load extremes are 2,960 and 2,156 kN-m, respectively, for the stall- and pitchregulated machines. The coefficient of variation based on the 1000 resampled median extremes decreases rapidly with the number of simulations such that it is only about $0.5 \%$ when 100 simulations are carried out. These results reconfirm that the uncertainty in the median extreme OOPB load from the turbine simulations is very small. In order to determine the number of simulations required to obtain stable median extreme estimates, a varying number of simulations $(1,2, \ldots, 100)$ are considered and the corresponding distribution of the resampled median extreme distribution is estimated. Figure 6 shows mean values and $90 \%$ confidence intervals on the median extreme OOPB load estimated for different numbers of simulations. The dashed lines represent $5 \%$ deviation from the mean value corresponding to the 100 -simulation case. It is seen that by using approximately 5-10 simulations, much of the sample falls within the $5 \%$ deviation. Since the EC method relies on stable estimates of the median extreme load, this suggests that for both turbines, the method can conveniently use only a small number of simulations for each inflow combination considered and design load estimates thus derived will be quite accurate.

\section{F. Comparison of Design Loads from the EC Method with those from the Parametric Method}

Out-of-plane bending (OOPB) design loads are now compared to those derived using a full integration approach that employs a parametric model for the short-term response distribution. ${ }^{8}$ In that study, inflow/turbine simulations were carried out over a range of $V$ values between 5 and $25 \mathrm{~m} / \mathrm{s}$ (in $1 \mathrm{~m} / \mathrm{s}$ increments) and $\sigma$ values between 0.2 and $5 \mathrm{~m} / \mathrm{s}$ (in $0.2 \mathrm{~m} / \mathrm{s}$ increments). Nine simulations per wind condition were performed, resulting in a total 4,725 simulations. A parametric model using statistical moments of 


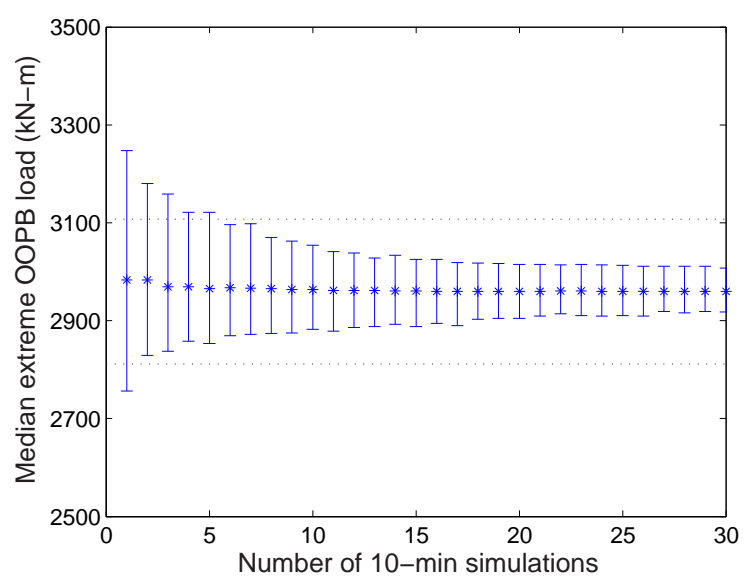

(a) Stall-regulated

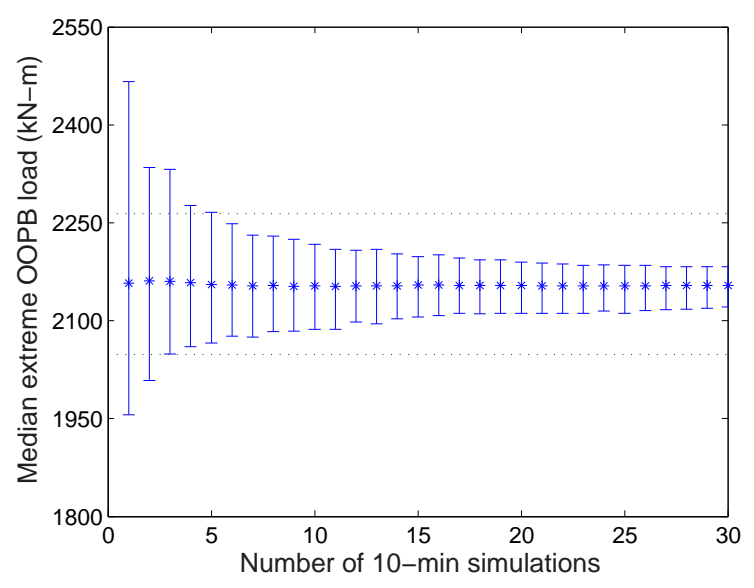

(b) Pitch-regulated

Figure 6. Mean values and $90 \%$ confidence intervals on median ten-minute extreme OOPB loads for the stalland pitch-regulated turbines based on different numbers of ten-minute simulations.

the short-term response (conditional on inflow variables, $V$ and $\sigma$ ) was fitted to the simulated turbine load maxima before carrying out the integration given by Eq. (1) numerically. Note that the "exact" probability distribution of turbine loads from a full year's worth of simulation was carried out by Moriarty et al, ${ }^{8}$ and can be used to evaluate the accuracy of the 1-year loads derived using both the EC and the parametric methods. For the stall-regulated machine, the EC method and the parametric method both predict similar OOPB design load levels even though the parametric model required 4,725 ten-minute turbine simulations while the EC method required only 90 simulations (corresponding to 5 simulations applied through the EC method's preliminary thirteen cases and four refined cases plus one additional case to that corrects for neglected response variability). In fact, for this stall-regulated turbine, the EC method's design load, after the preliminary search based on only 65 simulations, is almost the same as the final derived load after refinements. Note also that at the 1-year return period level, the EC method design load is consistent with the "exact" 1-year extreme load obtained from direct simulation by Moriarty et al. ${ }^{8}$ For the pitch-regulated machine, the EC method yields lower OOPB loads than the parametric method. At the 1-year level, however, the EC method's OOPB design load $(2,166 \mathrm{kN}-\mathrm{m})$ is much closer to the "exact" load of about 2,200 $\mathrm{kN}-\mathrm{m}$. This is in contrast to the significantly overpredicted design load of approximately $2,700 \mathrm{kN}-\mathrm{m}$ by the parametric method. The parametric method has difficulty in modeling the short-term OOPB loads for the pitch-regulated machine because of the complex behavior introduced by the control system. The EC method, however, is able to derive design loads even for this turbine quite accurately based on the 1-year return period load results. In summary, the EC method is able to predict blade-root OOPB design loads fairly well for both the stall-regulated and the pitch-regulated full-sized 1.5MW turbines studied.

Table 3. Comparison of estimated 1-year and 20-year extreme bending loads of the stallregulated and pitch-regulated machines in $\mathrm{kN}-\mathrm{m}$ based on 3 different probabilistic approaches.

\begin{tabular}{ccccc}
\hline & & \multicolumn{3}{c}{ Probabilistic approaches } \\
\cline { 3 - 5 } Turbine & $T(\mathrm{yrs})$ & $\mathrm{EC}^{+}$ & Parametric method $^{8}$ & "Exact" 8 from \\
& & $(18 \times 5$ simulations $)$ & $(4,725$ simulation $)$ & a full year's simulation \\
\hline \multirow{2}{*}{ Stall } & 1 & 2,971 & $\sim 3,000$ & $\sim 3,000$ \\
& 20 & 3,156 & $\sim 3,200$ & - \\
\hline \multirow{2}{*}{ Pitch } & 1 & 2,166 & $\sim 2,700$ & $\sim 2,200$ \\
& 20 & 2,326 & $\sim 2,900$ & - \\
\hline
\end{tabular}




\section{Conclusions}

The Environmental Contour (EC) method has been applied for predicting 20-year out-of-plane bending (OOPB) design loads at the blade root of two full-size 1.5MW turbines - one stall-regulated, the other pitchregulated. Results suggest that design loads predicted with the EC method are stable despite using only a small number of simulations (five) at each wind condition as well as a small number of wind conditions (thirteen) along the 20-year return period contour. The uncertainty of the extreme load statistics used by EC method to predict design loads was investigated. It was found that the uncertainty in load statistics, such as the ten-minute median extremes, is rather small when compared with the statistical moments of maxima commonly used with parametric methods in load extrapolation.

Since the EC method ignores response uncertainty, a correction factor was proposed to refine the design loads. This correction is based on the use of a locally fit lognormal model to extreme statistics and does not require extensive computations to derive the short-term response conditional on inflow random variables. No significant changes to the design loads from the EC method resulted in the cases studied when this refinement was applied.

Turbine design loads from the EC method were compared to those from a parametric method that requires a very large number of simulations to perform a load extrapolation leading to the desired design load. For the stall-regulated machine, the design loads from both methods were quite similar. However, for the pitch-regulated machine, the 1-year predicted load from the EC method was a lot closer to the "exact" value (available from simulation of one full year of load statistics) than that from the parametric method. The parametric method fails to accurately model the short-term OOPB load of the pitch-regulated machine because of the complex behavior introduced by the control system. The EC method, however, is able to yield design loads that are more reasonable for the pitch-regulated turbine and this was verified again with the 1-year design load.

In summary, the results presented here for two 1.5MW turbine models suggest that the EC method can be very useful for predicting accurate long-term design loads for wind turbines without requiring excessive computational effort.

\section{Appendix}

\section{A. Truncated Rayleigh Cumulative Distribution Function for $V$}

The cumulative distribution function for the ten-minute average Rayleigh wind speed, $V$, when limited to wind speeds between the cut-in wind speed, $v_{i}$, and cut-out wind speed, $v_{o}$, is

$$
F_{V}(v)=\frac{G\left(v_{i}\right)-G(v)}{G\left(v_{i}\right)-G\left(v_{o}\right)}
$$

where $G(v)=\exp \left(-\left(\frac{v}{\alpha}\right)^{2}\right)$. Note that the value of $\alpha$ is defined in Eq. (3).

\section{B. Parameters of $f_{\sigma \mid V}(v, \sigma)$}

The standard deviation, $\sigma$ of the ten-minute wind process conditional on the ten-minute wind speed, $V$, is assumed to follow a lognormal distribution. Hence, we have

$$
f_{\sigma \mid V}(v, \sigma)=\frac{1}{\sqrt{2 \pi \sigma} \zeta} \exp \left[-\frac{1}{2}\left(\frac{\ln \sigma-\lambda}{\zeta}\right)^{2}\right]
$$

where the mean, $\lambda$, and the standard deviation, $\zeta$, of $\ln \sigma$ are:

$$
\begin{aligned}
& \zeta=\sqrt{\ln \left(\delta_{\sigma \mid V}^{2}+1\right)} \\
& \lambda=\ln \mu_{\sigma \mid V}-\frac{1}{2} \zeta^{2}
\end{aligned}
$$

while $\delta_{\sigma \mid V}$, the conditional coefficient of variation of $\sigma$ given $V$ is:

$$
\delta_{\sigma \mid V}=\frac{\sigma_{\sigma \mid V}}{\mu_{\sigma \mid V}}
$$

$$
12 \text { of } 13
$$


The conditional mean and standard deviation of $\sigma$ given $V$ is provided per the IEC guidelines ${ }^{19}$ as follows:

$$
\begin{gathered}
\left.\mu_{\sigma \mid V}=\frac{I_{15}(15+a V)}{(a+1)}-2 I_{15} \quad \text { (in } \mathrm{m} / \mathrm{s}\right) \\
\sigma_{\sigma \mid V}=2 I_{15} \quad(\text { in } \mathrm{m} / \mathrm{s})
\end{gathered}
$$

where $I_{15}$ is the characteristic value of the turbulence intensity at $15 \mathrm{~m} / \mathrm{s}$, and $a$ is a slope parameter defined in the IEC guidelines. ${ }^{19}$ For a Class IA turbine site, as is the case assumed here, $I_{15}$ is 0.18 , and $a$ is 2 .

\section{Acknowledgments}

The authors gratefully acknowledge the financial support provided by Grant No. 30914 from Sandia National Laboratories and by a CAREER Award (No. CMS-0449128) from the National Science Foundation. They are also grateful to Dr. Patrick Moriarty of the National Renewable Energy Laboratory (NREL) for providing simulation models for the two turbines.

\section{References}

${ }^{1}$ Madsen, P. H., Pierce, K., and Buhl, M., "Predicting Ultimate Loads for Wind Turbine Design," Proceedings of the ASME Wind Energy Symposium, AIAA, Reno, NV, 1999, pp. 355-364.

${ }^{2}$ Larsen, D. C., Ronold, K. O., Jorgensen, H. E., Argyriadis, K., and de Boer, J., "Ultimate Loading of Wind Turbines,", Ris $\varnothing-R-1111$, Ris $\varnothing$ National Laboratory, Røskilde, Denmark, 1999.

${ }^{3}$ Ronold, K. O. and Larsen, G. C., "Reliability-based Design of Wind-turbine Rotor Blades against Failure in Ultimate Loading," Engineering Structures, Vol. 22, 2000, pp. 565-574.

${ }^{4}$ Fitzwater, L. M. and Winterstein, S. R., "Predicting Design Wind Turbine Loads from Limited Data: Comparing Random Process and Random Peak Models," Proceedings of the ASME Wind Energy Symposium, AIAA, Reno, NV, 2001, pp. 355-364.

${ }^{5}$ Manuel, L., Veers, P. S., and Winterstein, S. R., "Parametric Models for Estimating Wind Turbine Fatigue Loads for Design," Proceedings of the ASME Wind Energy Symposium, AIAA, Reno, NV, 2001, pp. 276-287.

${ }^{6}$ Fitzwater, L. M., Winterstein, S. R., and Cornell, C. A., "Predicting the Long Term Distribution of Extreme Loads from Limited Duration Data: Comparing Full Integration and Approximate Methods," Proceedings of the ASME Wind Energy Symposium, AIAA, Reno, NV, 2002, pp. 267-277.

${ }^{7}$ Moriarty, P. J., Holley, W. E., and Butterfield, S., "Effect of Turbulence Variation on Extreme Loads Predition for Wind Turbines," Proceedings of the ASME Wind Energy Symposium, AIAA, Reno, NV, 2002, pp. 278-287.

${ }^{8}$ Moriarty, P. J., Holley, W. E., and Butterfield, S. P., "Extrapolation of Extreme and Fatigue Loads using Probabilistic Methods," NREL/TP-500-34421, 2004.

${ }^{9}$ Peeringa, J. M., "Extrapolation of Extreme Responses of a Multi Megawatt Wind Turbine,", ECN-C-03-131, 2003.

${ }^{10}$ Cheng, P. W., van Bussel, G. J. W., van Kuik, G. A. M., and Vugts, J. H., "Reliability-based Design Methods to Determine the Extreme Response Distribution of Offshore Wind Turbines," Vol. 6, No. 1, 2003, pp. 1-22.

${ }^{11}$ Winterstein, S. R., Ude, T. C., Cornell, C. A., Bjerager, P., and Haver, S., "Environmental Contours for Extreme Response: Inverse FORM with Omission Factors," Proceedings of the ICOSSAR-93, Innsbruck, 1993.

${ }^{12}$ Fitzwater, L. M., Cornell, C. A., and Veers, P. S., "Using Environmental Contours to Predict Extreme Events on Wind Turbines," ASME Wind Energy Symposium, Reno, NV, 2003, pp. 244-258.

${ }^{13}$ Saranyasoontorn, K. and Manuel, L., "Efficient Models for Wind Turbine Extreme Loads using Inverse Reliability," Journal of Wind Engineering and Industrial Aerodynamics, Vol. 92, 2004, pp. 789-804.

${ }^{14}$ Madsen, H. O., "Omission Sensitivity Factors," Structural Safety, Vol. 5, 1988, pp. 35-45.

${ }^{15}$ Ronold, K. O., Wedel-Heinen, J., Christensen, C. J., and Jorgensen, A., "Reliability-based Calibration of Partial Safety Factors for Design of Wind Turbine Rotor Blades against Fatigue," Proceedings 5th European Wind Energy Conference, Thessaloniki, Greece, 1994.

${ }^{16}$ Veers, P. S. and Winterstein, S. R., "Application of Measured Loads to Wind Turbine Fatigue and Reliability Analysis," Proceedings of the ASME Wind Energy Symposium, AIAA, Reno, NV, 1997, pp. 160-169.

${ }^{17}$ Winterstein, S. R. and Engebretsen, K., "Reliability-based Prediction of Design Loads and Responses for Floating Ocean Structures," International Conference on Offshore Mechanics and Arctic Engineering, Lisbon, 1998.

${ }^{18}$ Rosenblatt, M., "The Spectrum of Hori-zontal Gustiness near the Ground in High Winds," Quart. J. Roy. Met. Soc., Vol. 23, 1952, pp. 470-472.

${ }^{19}$ IEC, "Wind Turbine Generator System Part 1: Safety Requirements," IEC/TC 88 61400-1, International Electrotechnical Commission (IEC), $2^{\text {nd }}$ Ed., 1998.

${ }^{20}$ Malcolm, D. J. and Hansen, A. C., "WindPACT Turbine Rotor Design Study," NREL/TP-500-34593, Global Energy Concepts LLC and Windward Engineering for the National Renewable Energy Laboratory, Golden, CO, August, 2002.

${ }^{21}$ Buhl, M. L., "SNwind User's Guide," National Renewable Energy Laboratory, Golden, Colorado, 2003.

${ }^{22}$ Buhl, M. L., Jonkman, J. M., Wright, A. D., Wilson, R. E., Walker, S. N., Heh, P., "FAST User's Guide," NREL/EL500-29798, National Renewable Energy Laboratory, Golden, Colorado, 2002.

${ }^{23}$ Efron, B. and Tibshirani, R. J., An Introduction to the Bootstrap, Chapman \& Hall, 1993. 